Musées, Patrimoine et Culture scientifiques et techniques

$115 \mid 2008$

janvier - février 2008

\title{
Désinsectiser les collections
}

\section{Michèle Gunn}

URL : http://journals.openedition.org/ocim/282

DOI : 10.4000/ocim.282

ISSN : 2108-646X

\section{Éditeur}

OCIM

Édition imprimée

Date de publication : 1 février 2008

Pagination : 15-22

ISSN : 0994-1908

Référence électronique

Michèle Gunn, «Désinsectiser les collections », La Lettre de I'OCIM [En ligne], 115 | 2008, mis en ligne le 10 novembre 2010, consulté le 20 avril 2019. URL : http://journals.openedition.org/ocim/282 ; DOI

$10.4000 /$ ocim. 282 
- le conditionnement,

- le transfert vers les lieux de stockage temporaire.

L'ensemble de ces opérations appelées « chantier des collections ", a été géré par un logiciel de gestion des collections, The Museum System (TMS), qui utilise des codes barres pour assurer la traçabilité des objets à chaque étape et à chaque mouvement. Le chantier des collections du musée du quai Branly s'est déroulé d'octobre 2001 à septembre 2004 dans l'immeuble industriel Le Berlier à Paris.

L'ampleur et la nature composite de la collection engagée dans cette chaîne, requerraient la mise en place de protocoles appropriés pour pouvoir tout traiter dans le temps imparti. Aussi a t-on jugé judicieux d'organiser ce chantier en deux campagnes.

La première campagne s'est déroulée d'octobre 2001 à juillet 2002. Celle-ci a permis de mettre en place, d'ajuster et d'éprouver les dispositifs et les protocoles nécessaires au bon déroulement des opérations. Cette première tranche a fait l'objet d'une évaluation par un comité constitué de responsables d'institutions patrimoniales et de logisticiens. Le comité s'est réuni trois fois avec les différents acteurs du chantier. À la suite des auditions, le comité a dressé un bilan qui a permis de dégager les points forts, les faiblesses et les potentialités liées à chaque étape. Une réflexion sur les améliorations a pu ainsi être engagée pour pouvoir tenir le délai des 3 ans (Naffah, C.)

La deuxième campagne a eu lieu d'octobre 2002 à septembre 2004, soit deux ans, en intégrant les modifications recommandées par le comité d'évaluation, notamment pour le traitement par anoxie. Ainsi, ce traitement qui au départ constituait l'étape $\mathrm{n}^{\circ} 1$ a été basculé en fin de circuit. Ainsi après leur emballage, les objets ont été soumis à l'anoxie avant d'être acheminés vers leurs lieux de stockage provisoire avant transfert au musée du quai Branly.

\section{L'anoxie comme choix de traitement systématique}

Les inspections de l'état sanitaire des collections dans les institutions d'origine par des experts ont montré l'existence d'une infestation par divers insectes (Renard, A.). Dans la mesure où il est difficile de retracer l'historique des dégradations microbiologiques et les mesures prises pour y remédier, le choix a été fait de traiter systématiquement tout objet comportant des matériaux organiques, par privation d'oxygène, utilisant l'anoxie dynamique par atmosphère modifiée d'azote. Ce choix est motivé d'abord par le fait que l'azote étant un gaz chimiquement inerte, le traitement peut s'appliquer à tout type de matériaux sans discrimination et ne nécessite pas un emballage spécifique ; ce n'est pas le cas avec les méthodes de traitement qui utilisent des gaz à effet toxique tel que l'oxyde d'éthylène (oxirane) par exemple ou des rayonnements électromagnétiques comme les micro-ondes ou les rayons gamma ou des méthodes basées sur des techniques de pointe comme le plasma ${ }^{(2)}$. Bien que possédant l'avantage d'être à triple action, fongicide, bactéricide et insecticide, la mise en œuvre du traitement à l'oxyde d'éthylène, se révèle délicate, du fait de sa haute toxicité d'une part et d'autre part de son inadéquation avec certains matériaux ; comme les composites organique-métallique et certains insecticides. En effet l'oxyde d'éthylène n'admet pas les objets ayant subi de précédents traitements à base de produits halogénés, notamment chlorés (voir le protocole du centre technique de la $\mathrm{BNF}$ ), ce qui était le cas de la plupart des objets de la collection. De plus les emballages majoritairement retenus sur le chantier, boîtes en plastique et chariots en métal ne se prêtent pas non plus à ce traitement. Les rayons gamma sont très ionisants pour les matériaux, c'est pourquoi leur utilisation n'est pas systématisée. De plus ce traitement ne peut être réalisé que par une société spécialisée.

La deuxième motivation de la systématisation était que les objets traités ne retournant pas dans leur emplacement d'origine mais dans un nouveau musée,

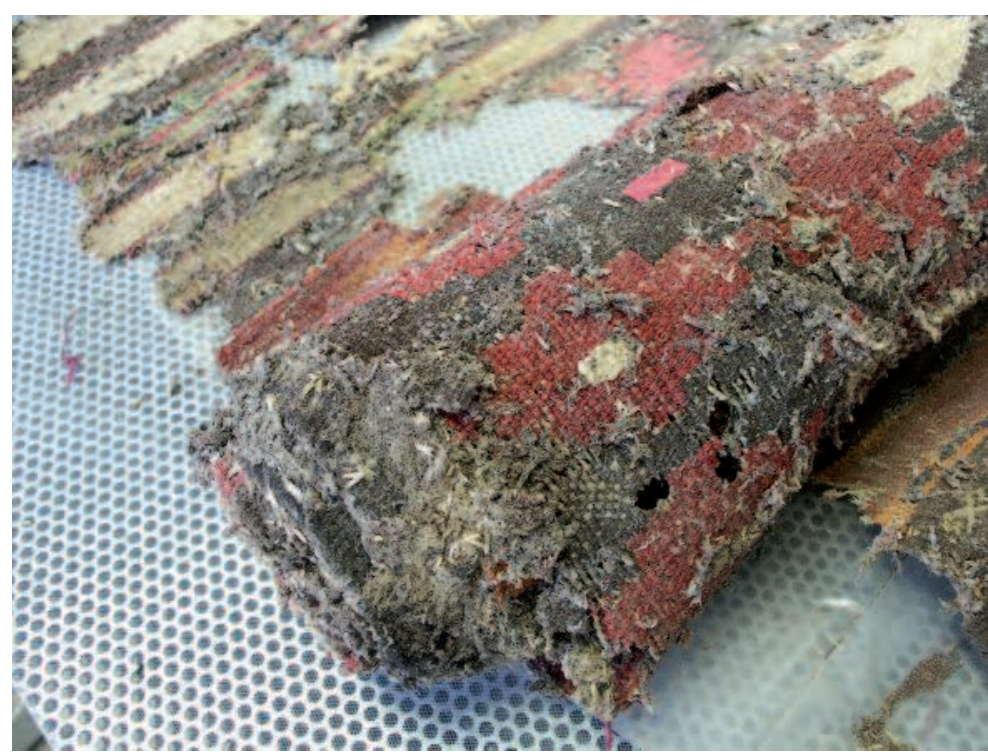

Un tapis infesté par des mites (c) M. Gunn 
un traitement de l'ensemble de la collection constituait une « remise à zéro » de l'état d'infestation et permettait d'envisager une traçabilité de l'état d'infestation des objets.

L'évaluation des risques de dégradation (effets secondaires) contre l'efficacité du traitement (effet curatif) ajoutée à la plus ou moins grande facilité de mise en œuvre sont les critères à croiser pour établir le choix du traitement lors d'un chantier des collections à flux tendu.

Toutes ces considérations font de l'anoxie une technique facile à intégrer dans une chaîne de traitement. De plus celle-ci peut être effectuée par le personnel, avec les précautions liées aux risques d'hypoxie (taux d'oxygène inférieur à $21 \%$ ).

La durée de traitement par anoxie couramment pratiquée par des institutions patrimoniales est de 21 jours. Les conditions généralement préconisées sont un taux d'oxygène inférieur ou égal à $0,1 \%$, une température de $20^{\circ} \mathrm{C}$ et une humidité relative autour de $50 \%$. Il est à noter que le traitement par anoxie immobilisant les objets, pendant plus de 21 jours, constituait une étape limitant l'avancement, dans la cinétique de la chaîne de traitement. Ajouté à cela, l'ampleur de la collection (environ 300000 objets), fait qu'il fallait mettre en place une installation et des procédures qui pouvaient prendre en compte ces deux considérations.

Ainsi, le dispositif d'anoxie a été conçu spécialement pour répondre aux besoins du chantier des collections. La grande particularité de ce dispositif réside d'une part dans sa taille et dans l'utilisation d'enceintes rigides en acier inoxydable et d'autre part dans sa capacité à générer rapidement une anoxie presque totale. Il en résulte une réduction considérable du temps mis pour abaisser le taux d'oxygène de $21 \%$ à $0,1 \%(1000 \mathrm{ppm})$ soit un à deux jours contre une semaine ou plus en fonction des chargements. Pour remédier au ralentissement induit par l'anoxie, plusieurs mesures ont été mises en place ; d'abord, des mesures organisationnelles, ensuite des mesures scientifiques avec une étude visant à établir si une réduction du temps de traitement était envisageable ou non :

\section{- les mesures organisationnelles}

La première est appelée la "Déviation Anoxie » (DA). Les objets ne présentant pas de signe manifeste d'infestation allaient directement aux étapes suivantes sans passer par l'anoxie. Ils étaient ensuite mis en attente pour le traitement en anoxie. Cette organisation, qui faisait alterner objets traités et objets non traités, demandait une mise en œuvre un peu lourde. Il fallait, en effet, mettre en place une surveillance accrue du circuit de traitement des objets. Pour cela il fallait un piégeage plus serré des insectes, et un changement complet des revêtements des plans de travail après le passage des objets dit DA. Si la DA a permis un approvisionnement régulier des étapes suivantes, le risque d'infestation devenait important en alternant objets traités et objets non traités. Aussi, la DA n'était pas une solution adéquate pour une remise à zéro correcte de l'état sanitaire des objets.

La deuxième mesure a consisté à basculer l'anoxie en fin de chaîne sur le site de Berlier ; c'est la «Filière Organique ». La systématisation de l'anoxie à la fin du circuit de traitement semblait une meilleure réponse à la fois pour le gain de temps et pour la qualité de la remise à zéro de l'état sanitaire.

La troisième mesure a été de faire appel à des prestataires extérieurs pouvant traiter les collections dans les mêmes conditions que sur le site de Berlier, «Anoxie Externe ", en fin de chaîne.

Parallèlement, un planning serré a été mis en place avec un à deux chargements par semaine. Les objets arrivaient, des sites de prélèvement, par lot de traitement plus ou moins homogènes en matériaux, en taille ou en typologie. Ils étaient conditionnés dans des cartons pourvus d'orifices ou dans des boîtes à claire-voie et rangés sur des palettes en bois ou sur des chariots conçus sur mesure. À chaque palette ou chariot correspondait un code barre. La palette était alors enregistrée dans le TMS à l'aide d'une douchette laser située à l'emplacement de l'enceinte où devait se dérouler le traitement.

\section{- les mesures scientifiques : étude sur l'optimisation du temps de traitement}

Compte tenu des délais à respecter, le temps était hautement précieux. Aussi convenait-il d'analyser le temps consacré à chaque étape de la chaîne de traitement. Pour cela un travail de recherche a été mené en parallèle avec le traitement des objets pour optimiser les conditions de traitement. Ce travail s'est appuyé sur une expérimentation sur des insectes et sur la diffusion de l'azote dans les matériaux. Les principaux résultats sont présentés ici avec pour objectif d'apporter une réponse aux diverses questions qui nous ont été posées à savoir : les procédures d'expérimentation sur les insectes, l'appauvrissement de l'oxygène à l'intérieur des objets et la résistance des insectes à l'anoxie et leur éventuelle mutation vers des espèces non « anoxiables». 


\section{Le traitement par anoxie sur le chantier des collections}

À la première campagne, après l'anoxie, le lot d'objets était défait aux étapes suivantes ; chaque objet recevait ensuite un code barre qui lui était propre. À la fin du circuit, les objets étaient conditionnés à nouveau sur des palettes également pourvues de codes barres et mis en stockage provisoire. À la deuxième campagne, avec l'anoxie en fin de circuit, les objets arrivaient dans leur conditionnement définitif de stockage provisoire.

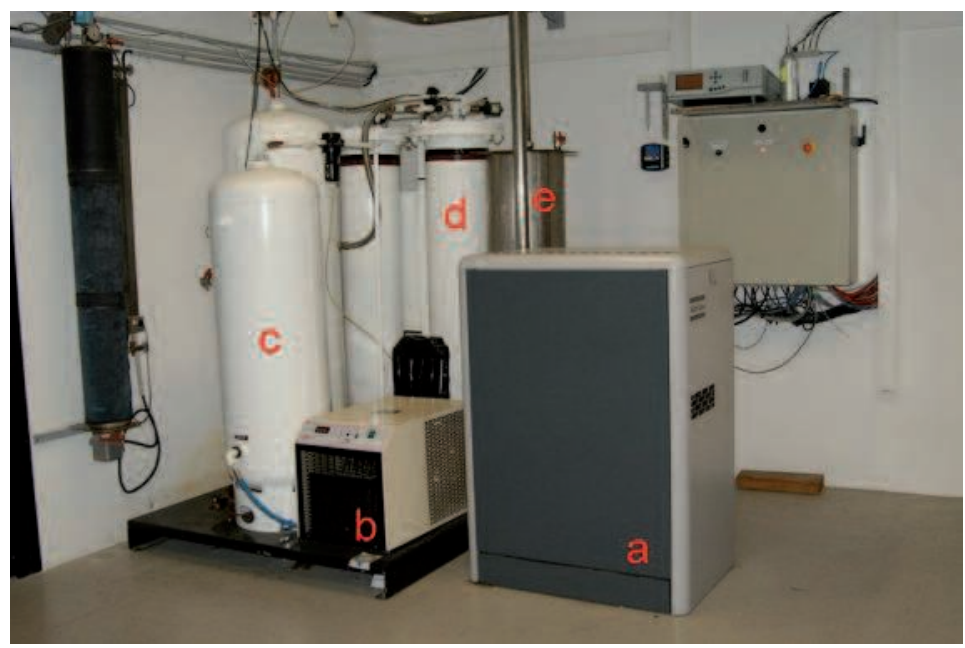

L'unité de production d'azote est composée d'un compresseur (a), d'un sécheur d'air (b), d'un réservoir d'air comprimé (c), de cuves de tamis moléculaires (d) et d'un réservoir d'eau pour l'humidification de l'azote (e). (c) M. Gunn

\section{Un équipement adapté à la mission}

\section{Description du dispositif}

Le dispositif d'anoxie est constitué de cinq parties : 1) une unité de préparation de l'azote, fourni par la société Technic Air ; 2) une unité de stockage de l'azote avec quatre réservoirs de $3 \mathrm{~m}^{3}$ chacun ;3) une unité de traitement avec trois enceintes $\mathrm{A}, \mathrm{B}$ et $\mathrm{C}$ de volume $25 \mathrm{~m}^{3}$ (B), et $35 \mathrm{~m}^{3}$ (A et C), 4) une unité de pilotage automatique et de supervision informatique, 5) une unité de contrôle du taux d'oxygène incluant un analyseur de traces d'oxygène Xentra, de la marque Servomex, pour les enceintes et deux détecteurs d'oxygène Oldham pour le local (3) :

- l'unité de préparation d'azote : le procédé de préparation d'azote, technique mise au point par la société Carbotrade, consiste à séparer sous pression de 7 Bars, l'azote de l'oxygène de l'air, par des tamis moléculaires. Le rendement est optimal avec un taux résiduel d'oxygène de 35 à 10 ppm.
- l'unité de traitement : au nombre de trois, les enceintes de traitement sont construites en acier inoxydable. Elles permettent de loger ensemble 32 palettes de dimensions standard $(80 \times 120 \mathrm{~cm})$. Les dimensions des trois enceintes sont adaptées à celles des palettes ou des chariots, afin d'éviter des volumes morts. Elles sont pourvues chacune de capteurs de température, d'humidité, de pression, et d'une prise de prélèvement de gaz pour l'analyse du taux d'oxygène résiduel.

- le système de supervision : les étapes du cycle de traitement sont contrôlées via l'ordinateur grâce à un jeu d'écran dont les accès sont réglementés par des codes. L'ensemble des opérations du cycle est pris en charge par un automate programmable logé dans la boîte électrique.

\section{Fonctionnement du dispositif}

Les consignes de traitement sont saisies au clavier dans un fichier prévu à cet effet. Exemple : taux d'humidité $50 \%$, temps d'exposition 21 jours (temps standard) ou autre durée, taux d'oxygène visé $0,1 \%$. Ces valeurs étaient modifiables. La température était contrôlée mais sa valeur de consigne ne pouvait être modifiée. La température des enceintes était fonction de celle de la salle ; celle-ci a été fixée à $25^{\circ} \mathrm{C}$. L'humidité relative dans les enceintes était régulée via l'humidification de l'azote, la consigne était fixée à $50 \%$. Le traitement comporte trois phases avec des appellations spécifiques :

- injection Gaz : c'est la phase de purge. Le taux d'oxygène est amené progressivement de $21 \%$ à $0,1 \%$. Cette période constitue le temps d'inertage (Ti). Celui-ci dépend des volumes des enceintes et de la nature du chargement. Pour initier la descente du taux d'oxygène, les enceintes sont balayées par $84 \mathrm{~m}^{3}$ d'azote pendant environ 1 heure.

- contact Gaz : la phase de traitement. Le taux d'oxygène est inférieur ou égal à $0,01 \%$. Dès que le taux d'oxygène atteint $0,1 \%$, le décompte du temps de traitement s'enclenche. Cette période correspond à la durée réelle de traitement;

- fin traitement : c'est la phase dite de rinçage. L'oxygène remonte à $21 \%$ par aspiration de l'air de la salle et évacuation de l'azote contenu dans l'enceinte à l'extérieur du bâtiment, à une hauteur qui ne met pas en danger la vie humaine.

Au cours du traitement tous les paramètres (température, humidité, taux d'oxygène, pression, temps de traitement) sont enregistrés et archivés dans des fichiers accessibles directement et exploitables via le logiciel Excel. À la fin du traitement, un rapport retraçant l'historique des événements est émis. 


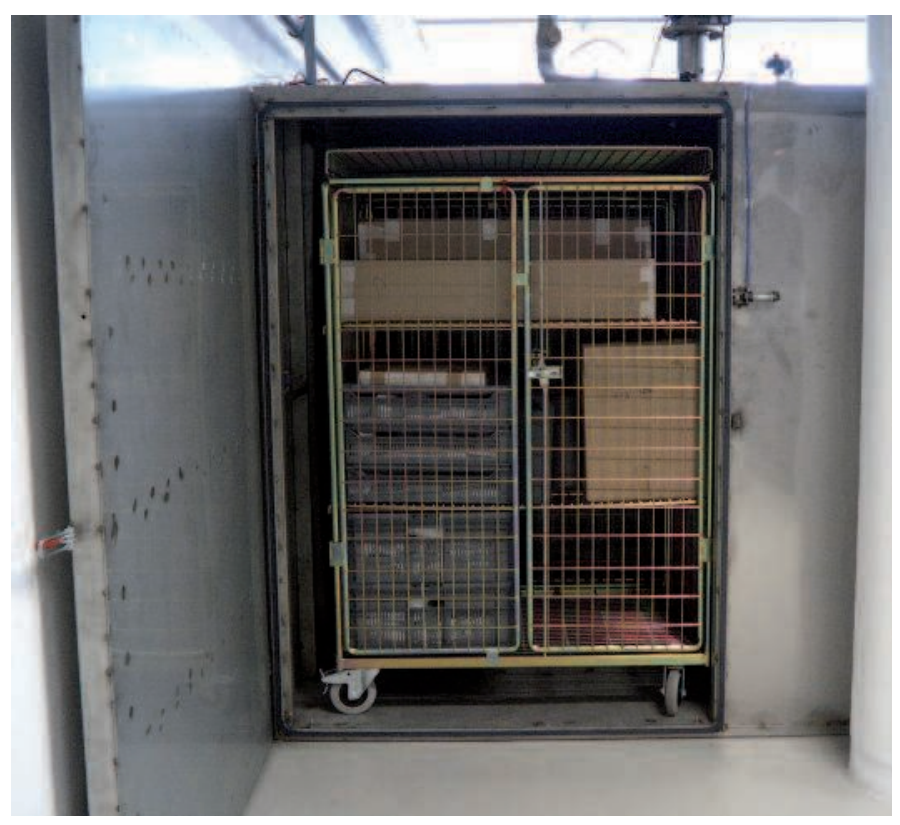

Vue d'un chargement : les objets étaient conditionnés avec du papier de soie et mis dans des boîtes en cartons pourvues d'ouvertures ou dans des boîtes en claire-voie.

(๑) M. Gunn

\section{À la recherche \\ du temps de traitement adéquat}

Chaque dispositif d'anoxie possède ses caractéristiques propres. Ainsi, le dispositif spécialement conçu pour le chantier des collections, que nous nommons le dispositif EPMQB (Établissement Public musée du quai Branly), étant nouveau et sans précédent dans le monde patrimonial de par sa taille et sa technique, il est nécessaire de mener une étude d'optimisation des conditions de traitement, notamment sur les temps d'inertage et les temps de traitement (temps d'exposition). Le but de cette étude n'est pas de refaire la démonstration de la faisabilité du traitement de l'infestation des objets par privation d'oxygène (Rust, M. et al, 1996 ; Selwitz, C. ; Maekawa, S., 1998). Il s'agit ici, de déterminer :

- le temps d'inertage, Ti, défini comme le temps d'abaissement du taux d'oxygène jusqu'à la valeur désirée dans l'enceinte, et de voir l'influence du chargement sur celui-ci avec le dispositif EPMQB ; - le temps d'exposition, Te, qui provoque $100 \%$ de mortalité à tous les stades de développement des insectes présents dans les objets avec le nouveau dispositif de traitement EPMQB, dans les conditions suivantes : une atmosphère très appauvrie en oxygène, entre $500 \mathrm{ppm}$ et $10 \mathrm{ppm}$, une température de $25^{\circ} \mathrm{C} \pm 1{ }^{\circ} \mathrm{C}$ et une hygrométrie de $50 \% \pm 5 \%$. Elles

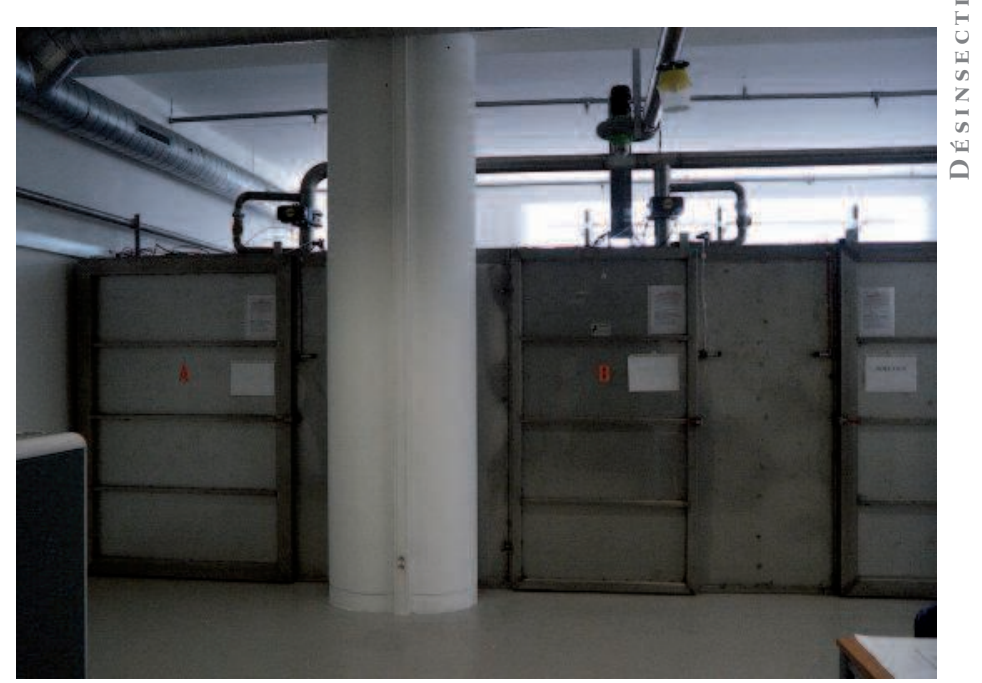

Les trois enceintes de traitement (volume de l'ensemble: $95 m^{3}$ répartis en $2 \times 35 m^{3}+25 m^{3}$ )

() M. Gunn

ont été choisies de façon optimale sans mettre en danger l'intégrité matérielle des objets ;

- le temps de désorption en oxygène des objets $\mathrm{Td}$. Celui-ci est intrinsèque au volume et aux matériaux constitutifs du chargement. Td est fonction de la perméabilité des matériaux vis-à-vis des gaz, ici, azote et oxygène.

\section{Méthodologie de l'étude}

Les données bibliographiques indiquent que les stades de développement les plus résistants sont ceux des œufs et des larves, les adultes et les nymphes sont les premiers à être inhibés par l'anoxie. Hylotrupes bajulus couramment appelé capricorne des maisons, s'est révélé être l'espèce la plus résistante à l'anoxie (Valentin, N.). Le capricorne n'est pas très courant dans les objets de musées. Il s'attaque généralement aux structures en bois résineux des bâtiments. Il a été choisi, ici pour sa résistance et pour sa rapide disponibilité : ces insectes sont en élevage au Centre Technique du Bois et de l'Ameublement (CTBA). En parallèle à l'expérimentation sur les insectes, d'autres expériences ont été réalisées pour mettre en évidence le temps de désorption de l'oxygène dans les matériaux, le degré d'appauvrissement en oxygène dans les matériaux et l'influence de la nature du chargement sur le temps d'inertage. 


\section{Expérimentation sur les insectes}

L'expérimentation a été réalisée sur des œufs et des larves de capricorne. Les éprouvettes ont été fournies par le Centre Technique du Bois et de l'Ameublement (CTBA). D'une manière générale, 50 à 60 œufs ont été déposés sur du papier buvard et conditionnés dans des boîtes de Petri ; 2 à 4 larves ont également été introduites dans des blochets en bois enfermés dans des boîtes de Petri.

Leétude comportait quatre séries d'expérimentations. Chaque série était constituée de trois expériences avec des durées de traitement identiques : d'abord 10 jours, c'est le temps minimal donné par la bibliographie (Valentin, N., 1993) ; puis 7 jours, ensuite 5 jours et finalement 14 jours. Chaque expérience était réalisée de la façon suivante : trois éprouvettes tests d'œufs et trois éprouvettes tests de larves étaient introduites dans l'enceinte de traitement en même temps que le chargement. Une éprouvette témoin d'œufs et une éprouvette témoin de larves étaient laissées dans l'ambiance du local. Cela représentait un total de 24 (3 x 8) échantillons par série. Après l'expérimentation, les éprouvettes ont été ré-expédiées au CTBA qui s'est chargé de les conditionner en étuve à $28^{\circ} \mathrm{C}$ pendant 15 jours pour le calcul du taux de mortalité.

Les quatre séries d'expérimentation ont été réalisées dans les conditions environnementales moyennes suivantes calculées à partir des fichiers des données enregistrées au cours des traitements : $25^{\circ} \mathrm{C} \pm 1^{\circ} \mathrm{C}, 50 \pm 3 \%$ avec des taux d'oxygène se situant entre 158 et 471 ppm $(0,0158 \%$ et $0,0471 \%)$. Le tableau 1 récapitule les résultats sur les taux de mortalité.

\begin{tabular}{|c|c|c|c|}
\hline \multirow[t]{2}{*}{ Expériences } & \multirow{2}{*}{$\begin{array}{l}\text { Temps d'exposition } \\
\text { (jours) }\end{array}$} & \multicolumn{2}{|c|}{ Taux de mortalité \% } \\
\hline & & CEufs & Larves \\
\hline \multirow[t]{2}{*}{ Série 1} & 10 & 100 & 100 \\
\hline & & \multicolumn{2}{|c|}{ Pas d'éclosion } \\
\hline \multirow[t]{2}{*}{ Série 2} & 7 & 100 & 100 \\
\hline & & \multicolumn{2}{|c|}{ Pas d'éclosion } \\
\hline \multirow[t]{3}{*}{ Série 3} & 5 & 64 & 100 \\
\hline & & \multirow{2}{*}{\multicolumn{2}{|c|}{$\begin{array}{l}\text { Éclosion après mise } \\
\text { en étuve à } 28^{\circ} \mathrm{C}\end{array}$}} \\
\hline & & & \\
\hline \multirow[t]{2}{*}{ Série 4} & 14 & 100 & 100 \\
\hline & & \multicolumn{2}{|c|}{ Pas d'éclosion } \\
\hline
\end{tabular}

Tableau 1 : Taux de mortalité en fonction du temps d'exposition Te
La série 1 a été traitée avec un temps d'exposition Te, de 10 jours : le tableau 2 montre la comparaison entre taux de mortalité par anoxie et taux de mortalité naturelle. Cette comparaison est nécessaire pour voir l'action réelle de la privation d'oxygène.

\begin{tabular}{|l|l|l|l|l|}
\hline \multirow{2}{*}{ Série 1 } & \multicolumn{2}{|l|}{$\begin{array}{l}\text { Éprouvettes } \\
\text { test sous azote } \\
\text { Taux de mortalité \% }\end{array}$} & \multicolumn{2}{|l|}{$\begin{array}{l}\text { Éprouvettes témoins } \\
\text { (dans le local) } \\
\text { Taux de mortalité \% }\end{array}$} \\
\cline { 2 - 5 } & CEufs & Larves & CEufs & Larves \\
\hline Expérience 1 & $\begin{array}{l}100 \\
\text { pas d'éclosion }\end{array}$ & 100 & $\begin{array}{l}0 \\
\text { éclosion, larves } \\
\text { en mouvement }\end{array}$ & 50 \\
\hline Expérience 2 & $\begin{array}{l}100 \\
\text { pas d'éclosion }\end{array}$ & 100 & $\begin{array}{l}\text { 0 éclosion, larves } \\
\text { en mouvement }\end{array}$ & 0 \\
\hline Expérience 3 & $\begin{array}{l}100 \\
\text { pas d'éclosion }\end{array}$ & 100 & $\begin{array}{l}0 \\
\text { éclosion }\end{array}$ & 25 \\
\hline
\end{tabular}

Tableau 2 : Taux de mortalité pour un temps d'exposition de 10 jours

Les résultats montrent qu'un temps d'exposition Te de 5 jours en anoxie dynamique n'est pas efficace à $100 \%$ pour tous les stades de développement. En effet si l'azote inhibe l'évolution des œufs vers le stade larvaire, $36 \%$ des œufs ont éclos après leur mise en étuve à $28^{\circ} \mathrm{C}$. Ainsi, la série 3 a été abandonnée dès la première expérimentation. À la suite de ce travail, le temps d'exposition retenu était de 7 jours. Par précaution, afin d'inclure des cas de désorption difficile, celui-ci a été multiplié par 2. Ainsi, le temps de traitement proprement dit, a été de 14 jours à partir de la deuxième campagne.

En ce qui concerne les temps mis pour atteindre l'anoxie dans les enceintes, chaque chargement a été analysé, objet par objet, à partir des fichiers TMS où sont inscrits des informations sur le matériau constitutif des objets, leurs dimensions, et leur poids. Dans tous les cas, il est apparu clairement que les enceintes de $35 \mathrm{~m}^{3} \mathrm{~A}$ et $\mathrm{C}$ atteignaient $0,1 \%$ d'oxygène de façon constante au bout d'une journée et demi ; quant à l'enceinte $\mathrm{B}$ de $25 \mathrm{~m}^{3}$, celle-ci ne requerrait qu'une journée. En revanche, il s'est avéré plus difficile de corréler les temps d'inertage avec la nature des chargements. La différence des temps 
d'inertage semble résider plus dans l'occupation de l'espace que dans la nature des matériaux. Lorsque le remplissage est maximal le temps d'inertage était plus long. Il a également été observé que les chargements constitués majoritairement d'objets en bois ou d'un mélange de bois, de textile et de peaux, demandaient des temps d'inertage plus long qu'un chargement de bois et de pâte végétale ou de bois et de fibres végétales.

La désorption de l'oxygène des matériaux a été mise en évidence par comparaison entre les temps d'inertage des enceintes chargées et les temps d'inertage des enceintes vides. Celle-ci est d'autant plus favorisée qu'il existe des fentes, des fissures, des galeries creusées par les insectes, en plus des microporosités naturelles des matériaux. Les résultats sont les suivants pour les enceintes de $35 \mathrm{~m}^{3}$ :

\begin{tabular}{|l|l|l|l|}
\hline Enceinte & $\begin{array}{l}\text { Ti moyen } \\
\text { avec chargement }\end{array}$ & $\begin{array}{l}\text { Ti moyen } \\
\text { sans chargement }\end{array}$ & $\begin{array}{l}\text { Td, temps } \\
\text { de désorption }\end{array}$ \\
\hline A & 33 heures $\pm 2 \mathrm{~h}$ & 23 heures & 10 heures $\pm 2 \mathrm{~h}$ \\
\hline $\mathrm{C}$ & 38 heures $\pm 2 \mathrm{~h}$ & 22 heures & 13 heures $\pm 2 \mathrm{~h}$ \\
\hline
\end{tabular}

Tableau 3 : Moyenne des Temps de désorption, Td

Les temps d'inertage obtenus, lorsque l'enceinte est vide, sont très constants. En revanche, les temps d'inertage, lorsque les enceintes sont chargées, varient en fonction du chargement. Pour montrer la désorption de l'oxygène des objets, des simulations ont été réalisées avec des boîtes en carton et en bois. Ces expériences ont montré qu'il y a bien une désorption d'oxygène des objets en anoxie dynamique, bien qu'il n'y ait pas de dépression initiale, comme c'est le cas en anoxie statique lors de la réaction chimique entre les absorbeurs et l'oxygène. Ce phénomène peut s'expliquer de la façon suivante : l'atmosphère des enceintes peut être considérée comme un mélange binaire, azote et oxygène. Les polluants hydrosolubles tels que le dioxyde de carbone $\left(\mathrm{CO}_{2}\right)$ et le dioxyde de soufre $\left(\mathrm{SO}_{2}\right)$ sont, en grande partie, éliminés avec l'eau dans le sécheur. La vapeur d'eau est une constante et à ce titre n'a pas d'influence sur l'échange gazeux oxygène/azote $\left(\mathrm{O}_{2} / \mathrm{N}_{2}\right)$. Le processus bien qu'étant dynamique, pourrait être décomposé en une succession d'états d'équilibre entre l'enceinte et le caisson (ou les objets). D'après le principe de modération de Lechâtelier, un système en équilibre, évolue d'un état d'équilibre à un autre état d'équilibre de façon à compenser la perturbation qui lui est imposée. Ici, la perturbation est la disparition de l'oxygène de l'enceinte. Lorsqu'un composé disparaît, l'équilibre est déplacé dans le sens qui favorise l'apparition de ce réactif. Lorsque l'enceinte s'appauvrit en oxygène l'équilibre est déplacé de façon à égaliser la concentration en oxygène. Le mouvement des molécules d'oxygène va naturellement dans le sens cartonenceinte : c'est le phénomène de désorption.

\section{Conclusion}

Seule l'anoxie dynamique peut raisonnablement être intégrée de façon systématique dans une chaîne de traitement lors d'un chantier des collections à flux tendu. La décision de traiter systématiquement ou non les œuvres doit s'appuyer sur une inspection au préalable de l'état sanitaire. La systématisation se justifie lorsque l'infestation est telle qu'il est difficile de distinguer collections saines et collections contaminées. Les simulations de désorption de l'oxygène faite avec des boîtes en bois et des palettes filmées (Gunn et al,) permettent de conclure que les conditions létales sont bien atteintes au cœur des objets. La désorption est d'autant plus rapide que le balayage en azote est constant. La succession de déséquilibres et d'équilibres entraîne une évolution du système vers des nouveaux états d'équilibres de plus en plus appauvris en oxygène, phénomène qui n'est pas réalisé dans le cas de l'anoxie statique. Ce mouvement perpétuel des gaz empêche l'installation d'un environnement statique et tranquille qui pourrait favoriser une éventuelle adaptation d'une larve enfouie dans l'objet. La question de l'accoutumance des insectes à une atmosphère modifiée et très appauvrie en oxygène est ici pertinente. En effet, l'étude a montré qu'un temps d'exposition de 5 jours, ne suffit pas pour éradiquer le stade d'œufs pour le capricorne des maisons. D'autres études ont montré que la survie des insectes sans oxygène n'est pas impossible mais très rare, et concerne surtout des insectes qui vivent en milieux aquatiques, dans l'eau ou dans la glace. C'est le cas de certains moucherons vivant dans l'eau. Ils sont capables de résister plus de 100 jours en absence d'oxygène par ralentissement de leur respiration, augmentation de leur réserve d'oxygène et par un changement physiologique qui leur permet d'éviter la dessiccation. Dans ces conditions très humides, même en anaérobiose, la dessiccation, cause première de la mort des insectes, ne se produit pas. L'adaptation des insectes à une atmosphère appauvrie d'oxygène n'est pas non 
plus un acquis quand celle-ci se produit. Elle est réversible sur les générations futures (Zebe, E.). Dans des conditions différentes des conditions aquatiques, c'est-à-dire dans l'agroalimentaire ou dans les institutions patrimoniales, une telle adaptation est peu probable car l'humidité relative est bien plus faible. Létude de la physiologie des insectes montre que les insectes sont en difficulté à partir de $0,5 \%$ (Hoback, W.-W.), un taux supérieur au taux d'oxygène toléré pour le traitement par anoxie.

Les paramètres tels que la température, l'humidité relative, et le taux d'oxygène qui permettent d'adopter une durée de traitement raisonnable et efficace sont actuellement bien établis. Le rôle de la température est capital. Aucune donnée bibliographique ne nous permet d'attester de l'efficacité des traitements à des températures inférieures à $20^{\circ} \mathrm{C}$. De même une réduction du temps de traitement n'est envisageable que lorsque la température est bien au-dessus de $22^{\circ} \mathrm{C}$ et après des tests. Les températures de traitement élevées permettent de conditionner les insectes au maximum de leur métabolisme ; ils respirent et prennent de l'azote au lieu de l'oxygène. Cela entraine une hyperventilation et une ouverture permanente des orifices respiratoires. Il en résulte la mort par dessiccation et par asphyxie. Le rôle de l'hygrométrie est moins marqué bien que le mécanisme principal de la mortalité soit la dessiccation aussi bien pour les larves que pour les insectes adultes.

Une collecte systématique des insectes trouvés morts ou vivants a été réalisée sur le chantier des collections. Ces insectes sont en cours d'identification. Un travail de corrélation entre le lieu, le matériau et l'espèce d'insecte, suivra l'identification.

\section{Notes}

(1) Il existe plusieurs techniques de traitement par anoxie : l'anoxie statique qui s'appuie sur la réaction chimique entre des absorbeurs d'oxygène qui sont des complexes de fer de l'oxygène de l'air. Contrairement aux idées reçues cette technique demande une mise en œuvre délicate. Comme toute réaction chimique, la réaction des absorbeurs avec l'oxygène ne peut se faire que dans des conditions bien spécifiques de concentration et de température. L'anoxie dynamique consiste en un balayage des enceintes par un gaz inerte, généralement de l'azote ; du dioxyde de carbone et quelquefois, de l'hélium et de l'argon sont utilités (Valentin, N., 1993). Il en résulte une atmosphère modifiée par dilution successive du taux d'oxygène jusqu'à obtention de l'anoxie. Ici le choix s'est porté sur l'azote car celui-ci peut être obtenu facilement à partir de l'air ambiant. Pour l'anoxie semi-dynamique, un balayage de la poche est effectué avec un gaz inerte puis celle-ci est scellée avec ou sans absorbeur. (2) Plasma : www.midwestfreezedryltd.com/Advanced $\% 20$ Plasma\%20 Sterilization.htm

micro ondes : www.cirad.fr/fr/prest_produit/materiel/page.php?id=47

(3) L'installation de l'ensemble du dispositif a été effectuée par la société Mallet. Ce premier dispositif était un prototype qui fait ses preuves, et depuis d'autres sociétés ont été équipées avec un dispositif similaire ; Hygiène-Office et Aïnu. Le musée du quai Branly est également équipé du même dispositif avec une capacité de traitement beaucoup moindre que sur le site du chantier des collections, soit quatre palettes. Il existe un autre dispositif, appelé VELOXY, né d'un projet européen qui permet de traiter par anoxie dynamique (celui-ci est actuellement commercialisé par la société Stouls).

\section{Bibliographie}

Gunn, M., Ziaeepour, H., Merizzi, F. et Naffah, C. Anoxia : treatment by oxygen deprivation. Optimising the museum object treatment time using the installation of collection treatment site of the public museum, musée du quai Branly, 2003 NOOX3 Conférence, 3 et 4 novembre 2003 (http://arxiv.org/ftp/physics/papers/0611/0611199.pdf)

Hoback, W.-W. et Stanley, D.-W. Insects in hypoxia, J. of Insects Physiology, 2001, n47, pp. 533-544.

Naffah, C., Musée du quai Branly, programme de conservation préventive, Paris 2004. Voir également La science au service de l'art et des civilisations : science et conservation, Techné, n²3, musée du quai Branly, 2006.

Renard, A. Étude complémentaire sur la désinfection des collections du musée de l'Homme et du musée national des Arts d'Afrique et d'Océanie. Rapport pour la Mission de Préfiguration du MHAC, janvier 2000.

Rust, M., Vinod, D., Druzik, J. et Presseur, F. The feasability of using modified atmosphere to control insect pests in museum, Restaurator, 1996, nº 17 , pp. 43-60.

Selwitz, C. et Maekawa, S. Inert gases in control of museum insects pests. Ed. The J. Paul Getty Trust, 1998.

Valentin, N. Comparative analysis of insect control by nitrogen, argon, and carbon dioxide in museum, archive and herbarium collection, International Biodeterioration and Biodegradation, 1993, n³2, pp. 263-278.

Valentin, N. et Preusser, F. Insect control by inert gases in museums archives and archives, Restaurator, 1990, $n^{\circ} 11$, pp. 22-33.

Zebe, E. Arthropods Insecta. Metazoan life without oxygen. New-York : Chapman Hall, 1991. 